\title{
A Weak Signal Detection Method Based on Double Time Difference of Arrival Collaborative Mechanism
}

\author{
Jia-Xu Yu, Zheng-Bo Sun, Qing He, Xin-Xin Ouyang \\ Science and Technology on Blind Signal Processing Laboratory, Chengdu, China, 610041 \\ E-mail: 943684129@qq.com
}

\begin{abstract}
Considering the weak signal of partial radiant point, the traditional TDOA(Time Difference of Arrival) detector cannot meet the requirement. A weak signal detection method based on double time difference collaborative mechanism is proposed. In this thesis, the double time difference collaborative detector is designed, and theoretical analysis and mathematical derivation of the performance of the detector is implemented. Then the processing gain is given out. At last, the simulation has confirmed the performance of the detector.
\end{abstract}

Keywords-correlation detection; weak signal detection; collaborative detection

\section{INTRODUCTION}

It is an important mission for the wireless sensors to manage to detect target under the low SNR circumstance.In recent years,as the development of new technic such as beam pointing control,gain control and so on,SNR of the signal radiated from target becomes weak, then the traditional detector failed to find the signal as the result.This leads to the need of finding new methods to detect target.

At last century 50s,some scholars proposed the matched filtering theory under maximum $\mathrm{SNR}^{[1-3]}$. The matched filtering is the best detector under WGN(White Gaussian Noise), however it requires the format of signals is totally known, which may not be sufficed in detection. And some scholars proposed the radiometer method to detect signals, which is also namely energy detector.This method can be used to adapt lots of signals cause it doesn't need priori informations so that it has been used widely. On the other hand,this method performance badly when the signal is weak.In recent years,some new weak signal detection methods are gradually proposed such as TBD(track-before-detection), chaos theory, wavelet transformation and so on.But all the methods aslo have many problems such as hard to apply engineering and so on.

The correlation detection is equivalent to matched filtering and can perform well to lots of signals,so many scholars from home and abroad have researched it deeply. The performance of correlation detection of LFM(Linear Frequency Modulation) signal is analyzed from [8],which find out the factors of the performance of correlation detection.An algorithm of correlation detection is presented to detect BOC signal of GPS from [9]. The block diagram of digital correlation detection system is designed from [10].

Although the correlation detection has been used in signal processing widely, this method still has the potential of improving its performance.Especially when the signal is weak,the correlation detector always fail to detect the
target.In this thesis, a double TDOA(Time Difference of Arrival) collaborative detector is presented, which improves the performance via using two pairs of TDOA stations collaborative working,to solve the weak LFM detection problem.Mathe-matical derivation of the theoretical model,test statistic and the threshold is implemented.The academic performance is analyzed and compared with the thraditonal correlation detection, then the processing gain is induced.At last the result of Monte-Carlo simulation confirms our conclusion.

\section{DESIGNING OF DOUBLE TDOA COLLABORATIVE DETECTOR}

\section{A. Theroetical Model}

We tend to use a pair of sensors as the TDOA stations under the traditional detection mechanism.We use a threshold to detect the correlation peak of the signal received by TDOA stations. The correlation peak tend to be a obvious value so that the detector can manage to find the signal under the high SNR circumstance, while the peak will be flooded with noise when the signal becomes weak,thus we cannot detect it.

To solve this problem,a method occurs to us easily,namely collaborative detection by multiple sensors. The peaks will be cumulated by the TDOA curves crossing at the position where the targets emerge,thus the detection performance can be improved,and the configuration is shown in Fig.1.

We assume the target transmits LFM signals, the complex envelope of the signal is

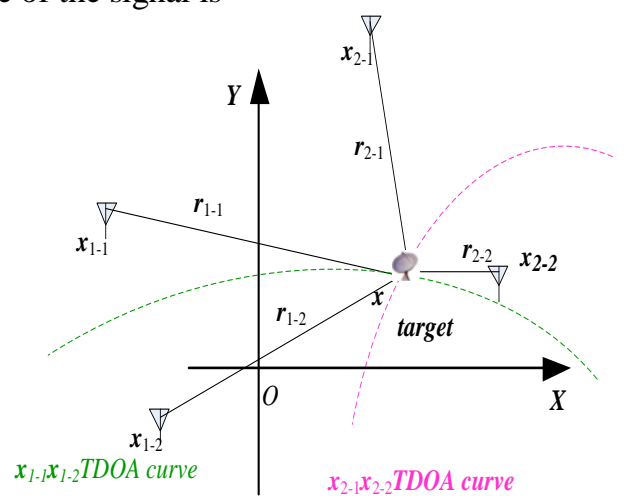

Figure 1. Physical configuration of double TDOA collaborative detection.

$$
S(t)=A e^{2 \pi f_{0}+2 \pi \frac{\mu}{2} t^{2}}
$$


Where $\mu=\frac{B_{0}}{T_{0}} \quad$ is the modulation rate, $B_{0}$ is the bandwidth, ${ }_{0}$ is the pulsewidth. Only single pulse will be considered here,so that $T_{0}$ is also the observation time.A is the ampl-itude, and the power is $P=\frac{A}{2}$.We assume that the noise at each of the receiver stations is WGN and the noise is independent from noise at other stations. The variance of noise is $\sigma_{n}^{2}$,so the noise will be denoted as $w \sim N\left(0, \sigma_{n}^{2}\right)$.And the SNR is

$$
S N R=10 \lg \frac{A}{2 \sigma_{n}^{2}}
$$

The hypothesis test for the detection problem can be written as follow

$$
\begin{cases}H_{0}: x(t)=w(t) & 0 \leq \mathrm{t} \leq \mathrm{T}_{0} \\ H_{1}: x(t)=s(t)+w(t) & 0 \leq \mathrm{t} \leq \mathrm{T}_{0}\end{cases}
$$

The null hypothesis would correspond to the situation of absence of signal,whereas the alternative hypothesis would be associated to the presence of signal.

\section{B. The Distribution of Test Statistic}

The initial problem is to establish the test statistic after we found the hypothesis test model. A pair of sensors are always used as the TDOA stations under the traditional detection mechanism, then We used to detect the correlation peak of the signal received by receiver stations.the correlation function is

$$
R_{s}(\tau)=\lim _{T_{0} \rightarrow \infty} \frac{1}{T_{0}} \int_{-\frac{T_{0}}{2}}^{\frac{T_{0}}{2}} s(t) s^{*}\left(t-\tau_{0}\right) d t
$$

Generally speaking,correlation detection performs well when SNR is $-6 \mathrm{~dB}$. While the correlation peak can be flooded with noise when the signal becomes weak. Thus the scheme of double TDOA collaborative detector is to manage to make the correlation peak above the noise by cumulating the two peaks. The correlation of signal of each pair receiver station is

$$
R_{s_{i}}(\tau)=\lim _{T_{0} \rightarrow \infty} \frac{1}{T_{0}} \int_{-\frac{T_{0}}{2}}^{\frac{T_{0}}{2}} S_{i}(t) s_{i}^{*}\left(t-\tau_{0}\right) d t, i=1,2
$$

However,the correlation peaks can't be directly calculated.At first we must transform the peaks onto TDOA curves on spatial domain,two TDOA curves will cross at the position where the target emerge, and that is also the theory of TDOA location. The correlation peaks will be cumulated here,then an obvious peak will appear,and the result of cumulating as shown in Fig.2:

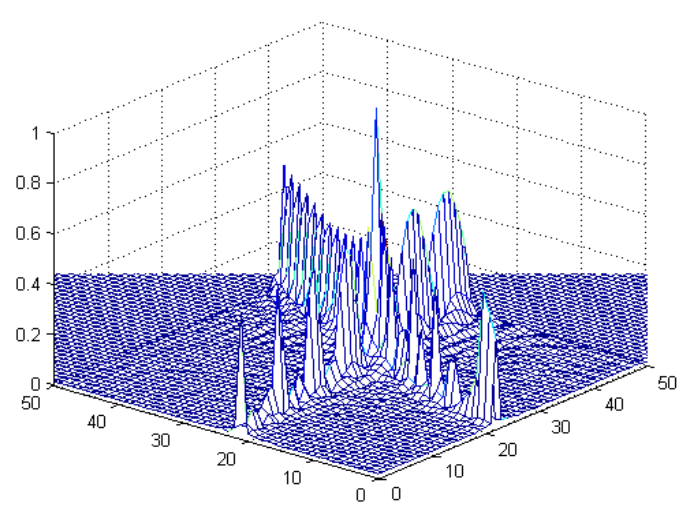

Figure 2. The cumulation of double TDOA correlation peaks.

From [10],mean of the correlation peak is $E\left(R_{\text {corr }}\left(K_{\tau_{0}}\right)\right)=\left(N-K_{\tau_{0}}\right) \sigma_{s}^{2}$, where $K_{\tau_{0}}$ means the TDOA after sampling,N stands for the sampling time, $\sigma_{s}^{2}$ means the variance of signal.And the variance of the noise after correlation is $D\left[R_{\text {corr }}\left(K_{\tau_{0}}\right)\right]=2\left(N-K_{\tau_{0}}\right) \sigma_{n}^{2} \sigma_{s}^{2}+N \sigma_{n}^{4}$.

According to the central-limit theorem,the correlation peak converges to a Gaussian distribution when the signal appears.So it can be denoted as $N\left(\left(N-K_{\tau_{0}}\right) \sigma_{s}^{2},\left(2\left(N-K_{\tau_{0}}\right) \sigma_{n}^{2} \sigma_{s}^{2}+N \sigma_{n}^{4}\right)\right.$.And when the signal is absent,the distribution can be denoted as $N\left(0, N \sigma_{n}^{4}\right)$

When two pairs of TDOA stations collaborate,both the peak and noise will be cumulated.Thus the distribution is $N\left(2\left(N-K_{\tau_{0}}\right) \sigma_{s}^{2}, 2 *\left[\left(2\left(N-K_{\tau_{0}}\right) \sigma_{n}^{2} \sigma_{s}^{2}+N \sigma_{n}^{4}\right]\right)\right.$.That is the distribution of test statistic.

\section{The Detection Threshold}

When the signal is absent,the noise on each mesh point is denoted as $N\left(0,2 N \sigma_{n}^{4}\right)$. When we detect the test statistic,we detect the maximum of $M$ mesh points actually.In other words, the maximum of $\mathrm{M}$ dependent Gaussian distribution random variables.According to [11],if $X_{1}, X_{2}, \cdots, X_{n}$ has a Gaussian distribution as $N\left(\mu, \sigma^{2}\right)$, the maximum of $X_{1}, X_{2}, \cdots, X_{n}$ has the distribution of extreme value I type.and the distribution function is 


$$
F_{Y_{n}}(y)=\exp \left(-\exp \left(-a_{n}\left(y-u_{n}\right)\right)\right)
$$

Where $u_{n}=\sigma u_{n}^{\prime}+\mu=\sigma \sqrt{2 \ln n}-\sigma \frac{\ln \ln n+\ln 4 \pi}{2 \sqrt{2 \ln n}}+\mu$,

$$
a_{n}=\frac{a_{n}^{\prime}}{\sigma}=\frac{\sqrt{2 \ln n}}{\sigma}
$$

maximum of the noise on the $\mathrm{M}$ mesh points is

$$
F_{Y_{M}}(y)=\exp \left(-e^{-a_{M}\left(y-u_{M}\right)}\right)
$$

$$
\begin{aligned}
& \qquad u_{M}=\sqrt{N} \sigma_{n}^{2} \sqrt{2 \ln M}-\sqrt{N} \sigma_{n}^{2} \frac{\ln \ln M+\ln 4 \pi}{2 \sqrt{2 \ln M}} \\
& \text { Where } \\
& { }_{a_{M}=\frac{\sqrt{2 \ln M}}{\sqrt{N} \sigma_{n}^{2}}} \text {,.so the false alarm probability is }
\end{aligned}
$$

$$
P_{f a}=\operatorname{Pr}\left(T>\gamma ; H_{0}\right)=1-F(\gamma)=1-\exp \left(-e^{-a_{M}\left(\gamma-u_{M}\right)}\right)
$$

the threshold is denoted as

$$
\gamma=u_{M}-\frac{\ln \left(-\ln \left(1-P_{F A}\right)\right)}{a_{M}}
$$

\section{PERFORMANCE OF THE DOUBLE TDOA COLLABORATIVE DETECTOR}

According to the conclusion of the former two section,the detection probability is

$$
P_{D}=Q\left(\frac{\gamma-2\left(N-K_{\tau_{0}}\right) \sigma_{s}^{2}}{\sigma_{1}}\right)
$$

Where

$$
\sigma_{1}=\sqrt{2\left[2\left(N-K_{\tau_{0}}\right) \sigma_{n}^{2} \sigma_{s}^{2}+N \sigma_{n}^{4}\right]}
$$

And the false alarm probability is denoted as

$$
P_{f a}=1-\exp \left(-e^{-a_{n}\left(\gamma-u_{n}\right)}\right)
$$

So the detection probability can be written as follow:

$$
P_{D}=Q\left(\frac{\gamma-2\left(N-K_{\tau_{0}}\right) \sigma_{s}^{2}}{\sigma_{1}}\right)
$$

$$
=Q \frac{\sigma_{2} * \sqrt{2 \ln M}-\sigma_{2} * \frac{\ln (\ln M)+\ln 4 \pi}{2 \sqrt{2 \ln M}}}{\sigma_{1}}-
$$$$
\left.\frac{-\sigma_{2} * \frac{\ln \left(-\ln \left(1-P_{f a}\right)\right)}{\sqrt{2 \ln M}}-2\left(N-K_{\tau_{0}}\right) \sigma_{s}^{2}}{\sigma_{1}}\right)
$$

$$
=Q\left(\frac{\sigma_{2}}{\sigma_{1}} *\left(\sqrt{2 \ln M}-\frac{\ln (\ln M)+\ln 4 \pi}{2 \sqrt{2 \ln M}}-\frac{\ln \left(-\ln \left(1-P_{f a}\right)\right)}{\sqrt{2 \ln M}}\right)\right.
$$$$
\left.-2 \frac{\left(N-K_{\tau_{0}}\right) \sigma_{s}^{2}}{\sigma_{1}}\right)
$$

Where $\sigma_{2}=\sqrt{N \sigma_{n}^{4}}$ is the standard deviation of the cumulated noise after correlation,in other words, it is the standard deviation of the test statistic under $H_{0}$. When the $S N R_{i}=\frac{\sigma_{s}^{2}}{\sigma_{n}^{2}}$ $N-K_{\tau_{0}} \approx N$,the last term in (12) can be denoted as

$$
\begin{aligned}
& 2 \frac{\left(N-K_{\tau_{0}}\right) \sigma_{s}^{2}}{\sigma_{1}}= \\
& 2 \frac{\left(N-K_{\tau_{0}}\right) \sigma_{s}^{2}}{\sqrt{2\left[\left(N-K_{\tau_{0}}\right) \sigma_{s}^{4}+2\left(N-K_{\tau_{0}}\right) \sigma_{n}^{2} \sigma_{s}^{2}+N \sigma_{n}^{4}\right]}} \\
& \approx 2 \frac{N}{\sqrt{2\left(N+2 N\left(\frac{\sigma_{n}}{\sigma_{s}}\right)^{2}+N\left(\frac{\sigma_{n}}{\sigma_{s}}\right)^{4}\right)}} \approx \sqrt{2 N} S N R_{i}
\end{aligned}
$$

At the same time,

$$
\begin{aligned}
& \frac{\sigma_{2}}{\sigma_{1}}=\sqrt{\frac{N \sigma_{n}^{4}}{2\left[2\left(N-K_{\tau_{0}}\right) \sigma_{n}^{2} \sigma_{s}^{2}+N \sigma_{n}^{4}\right]}} \\
& \sqrt{\frac{1}{2\left[2 \frac{N-K_{\tau_{0}}}{N} \frac{\sigma_{s}^{2}}{\sigma_{n}^{2}}+1\right]}} \approx \sqrt{\frac{1}{2}}
\end{aligned}
$$

Thus the detector performance is 


$$
\begin{aligned}
& P_{D}=Q\left(\frac { \sqrt { 2 } } { 2 } \left(\sqrt{2 \ln M}-\frac{\ln (\ln M)+\ln 4 \pi}{2 \sqrt{2 \ln M}}-\right.\right. \\
& \left.\left.\frac{\ln \left(-\ln \left(1-P_{f a}\right)\right)}{\sqrt{2 \ln M}}\right)-\sqrt{2 N} S N R_{i}\right)
\end{aligned}
$$

$N$ and $K_{\tau_{0}}$ are constant under each detection,so the detection probability changes with the SNR and is determinated by SNR, the number of TDOA curves, the number of mesh point $M$,the length of datas $N$,TDOA $\tau_{0}$.

\section{A. Processing Gain}

From [10],the output SNR is denoted as

$$
\begin{aligned}
& S N R_{o}=\frac{\left[E\left[R_{\text {corr }}\left(K_{\tau_{0}}\right)\right]\right]^{2}}{D\left[R_{\text {corr }}\left(K_{\tau_{0}}\right)\right]}=\frac{\left(N-K_{\tau_{0}}\right)^{2} \sigma_{s}^{4}}{2\left(N-K_{\tau_{0}}\right) \sigma_{s}^{2} \sigma_{n}^{2}+N \sigma^{4}} \\
& =\frac{N-K_{\tau_{0}}}{\frac{N}{N-K_{\tau_{0}}} \times \frac{\sigma_{n}^{4}}{\sigma_{s}^{4}}+2 \times \frac{\sigma_{n}^{2}}{\sigma_{s}^{2}}}=\frac{N-K_{\tau_{0}}}{\frac{N}{N-K_{\tau_{0}}} \times \frac{1}{S N R_{i}^{2}}+2 \times \frac{1}{S N R_{i}}}
\end{aligned}
$$

If the sample frequency is high, $N-K_{\tau_{0}} \approx N$, the SNR is

$$
S N R_{o}=\frac{N \times S N R_{i}^{2}}{2 S N R_{i}+1}
$$

Then the processing gain is

$$
P G=\frac{S N R_{o}}{S N R_{i}}=\frac{N \times S N R_{i}}{2 S N R_{i}+1}
$$

Under the double TDOA collaborative mechanism,the output SNR is

$$
S N R_{s o}=\frac{\left[E\left[2 R_{\text {corr }}\left(K_{\tau_{0}}\right)\right]\right]^{2}}{D\left[2 R_{\text {corr }}\left(K_{\tau_{0}}\right)\right]}=\frac{2 N \times S N R_{i}^{2}}{2 S N R_{i}+1}
$$

So the processing gain is

$$
P G=\frac{S N R_{s o}}{S N R_{i}}=\frac{2 N \times S N R_{i}}{2 S N R_{i}+1}
$$

\section{B. Simulation and Analysis}

Now we use Monte-Carlo simulation to testify our conclusion.
The bandwidth is $B=20 \mathrm{KHz}$, the cumulated time is $t=200 \mathrm{~ms}$.According to equation (9) and equation (12),we have the result as shown in Fig.3.A comparison of the double TDOA collaborative detector to traditional TDOA detector is given in Fig.4.D1 corresponds to the traditional detector and D2 corresponds to the double TDOA detector.According to the result of Fig.4,the double TDOA collaborative detector performs better.

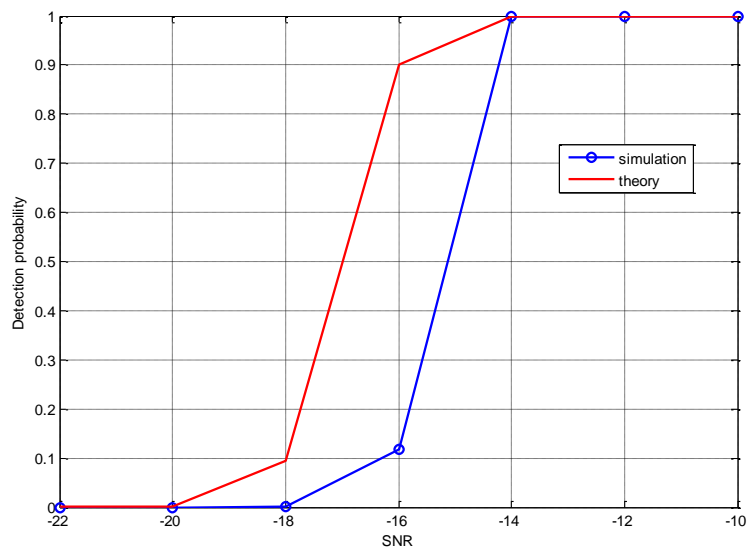

Figure 3. The detection performance under $P_{f a}=10^{-6}$.

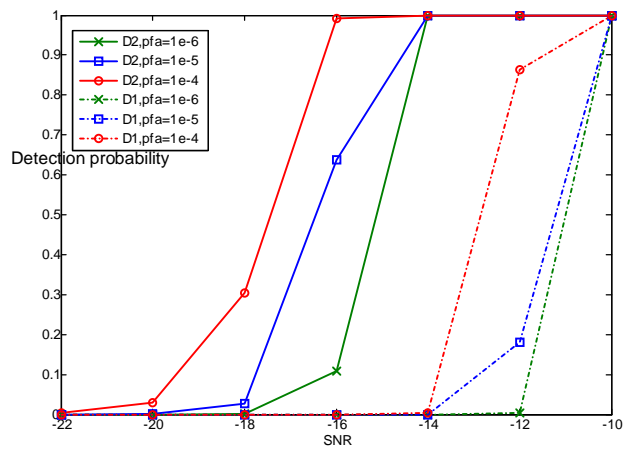

Figure 4. Detection performance of double TDOA collaborative detector against traditional TDOA detector.

\section{CONCLUSIONS}

In this paper, we have designed the double TDOA collaborative detector based on the traditional correlation detector to solve the weak signal detection problem. We have modeled the problem of detection and find out the test statistic under $H_{0}$ has distribution of extreme value I type,thus the threshold could be easily induced.The Monte-Carlo simulation result is consistent with the theory.The processing gain is $3 \mathrm{~dB}$ higher than the traditional method.

\section{REFERENCES}

[1] Skolnik M I. "Radar Handbook".3rd ed. New York, USA: McGraw-Hill, 1992. 
[2] Barton D K. "Radar System Analysis".Dedham,USA:Artech House, 1976.

[3] Steven M.Key. "Fundamentals of Statistical Signal Processing"Publishing House of Electronics Industry,2013.

[4] F.F.Digham,M.S.Alouini,M.K.Simon. "On the energy detection of unknown signals over fading channels".IEEE Transactions on Communications,2007,5.1:pp.21-24.

[5] O.Olabiyi,S.Alam,O.Odejide,et a1."Further Results on the Energy Detection of Unknown Deterministic Signals Over Generalized Fading Channel".IEEE Globecom Workshops,2011,pp.816-824

[6] S.P.Herath,N.Rajatheva,C.Tellambura."Energy detection of unknown signals in fading and diversity reception". IEEE Transactions on Communications, 2011,59(9):pp.2443-2453

[7] Kasun T. Hemachandra, Norman C. Beaulieu."Novel analysis for performance evaluation of energy detection of unknown deterministic signals using dual diversity". vol.4, IEEE Vehicular Technology Conference,2011.pp.2443-2453

[8] Yao Shan-feng,Yan Hang,Zeng An-jun,Hu Kuo."Correlation detection performance analysis for linear frequency modulation signal".vol.38 Computer Engineering,2012,01:pp.77-80.

[9] Qian Bo,Tian Hao-ruing,Feng Yong-xin. "Research on an algorithm of correlation detection for BOC modulation signal". Fire Control and Command Control,2011,04:pp.22-25.

[10] Huang Guigen,Li Pin,Ding Jian."Digital correlation detection and TDOA estimation of broadband jamming signal".Modern Radar,2015,05:pp.21-24.

[11] Yuan Zihou,He Xiaoya,Mei Jiabin."Distribution of maximum of mutilple independent Gaussian random variables".Journal of Wuhan University of Science and Engineering,2004,01:pp.52-55. 